\title{
Critical Exponents of the Two-layer Ising Model
}

\author{
Z. B. Li, ${ }^{1,2}$ Z. Shuai, ${ }^{3}$ Q. Wang, ${ }^{4}$ H.J. Luo, ${ }^{4}$ and L. Schülke ${ }^{4}$ 间 \\ ${ }^{1}$ Zhongshan University, Guangzhou 510275, China \\ ${ }^{2}$ Associate Member of ICTP, Trieste, Italy \\ ${ }^{3}$ Universitat de Mons-Hainaut, 7000 Mons, Belgium \\ ${ }^{4}$ Universität Siegen, D-57068 Siegen, Germany
}

\begin{abstract}
The symmetric two-layer Ising model (TLIM) is studied by the corner transfer matrix renormalisation group method. The critical points and critical exponents are calculated. It is found that the TLIM belongs to the same universality class as the Ising model. The shift exponent is calculated to be 1.773 , which is consistent with the theoretical prediction 1.75 with $1.3 \%$ deviation.
\end{abstract}

PACS numbers: 05.50.+q, 02.70.-c

Keywords: corner transfer matrix; two-layer; Ising; critical exponent

a. Introduction The two-layer Ising model (TLIM), as a simple generalisation of the twodimensional Ising model and a simple model for the magnetic ultra-thin films, has been studied for a long time [1, 2, 3, 4, 5, 6. Cobalt films grown on a $\mathrm{Cu}(100)$ crystal, for instance, have highly anisotropic magnetisation [7] and could therefore be viewed as layered Ising-spin systems. It has been found that capping $\mathrm{PtCo}$ in $\mathrm{TbFeCo}$ to form a two-layer structure has applicable features, for instance, raising the Curie temperature and reducing the switching fields for over-writable magneto-optical disks [8]. Apart from various possible applications to real physical materials, the model is theoretically interesting for its rich phase structure. The model has several interesting equivalents, such as a two-species gauge invariant Ising model [9], a spin- $\frac{3}{2}$ Ising model [10], a model of the dilute lattice gas and a quantum spin- $\frac{1}{2}$ ladder at zero temperature. The TLIM is important for the investigation of crossover from the 2-dimensional Ising model to the 3-dimensional one. In particular, it has been argued that the critical point of the latter could be found from the spectrum of the TLIM 11 .

In recent years, some approximation methods have been applied to this model 110, 12, 13, 14, 15, 16, 17, 18 . A critical line has been found in all these studies. As expected, the Curie temperature is very sensitive to the inter-layer interaction. Many discussions have been directed to the shift exponent at the decoupling point. Abe [3] and Suzuki [- 4 have predicted $\gamma=7 / 4$ for the isotropic model many years ago. Only in recent years the computational results have converged to the prediction with still about $2.3 \%$ deviation 16, 17.

\footnotetext{
* Supported by the National Natural Science Foundation of China under the project 19772074 and by the Deutsche Forschungsgemeinschaft under the project Schu 95/9-3.

${ }^{\dagger}$ Electronic address: LSchuelke@t-online.de
}

Apart from the shift exponent, it is also interesting to study the critical behaviour along the critical line. The model has the same critical exponents at the two ends of the critical line corresponding to the solvable decoupling limit and the infinite interlayer coupling limit. But it is clear that the decoupled system has a higher symmetry than the coupling layers, hence one cannot assume a priori that the TLIM belongs to the one-layer Ising universality class. Unusual finite-size effects have been observed in the dynamic process [19]. It has been proposed that the critical exponents (or some of them) would vary continuously along the critical line [9, 17]. However, there are also arguments in favour of unchanged exponents. Based on their correlation length equality method, Angelini et al. argued that the exponent $\nu$ is a constant [13. The accurate computation of critical exponents along the critical line remains a difficult unsolved problem.

It is our purpose to provide a reliable prediction for the critical exponents based on the corner transfer matrix renormalisation group (CTMRG) method [20, 21, 22].

The CTMRG method follows White's idea of density matrix renormalisation group 223, 24]. It reduces the phase-space dimensions by omitting the eigenstates corresponding to small eigenvalues of the density matrix. But instead of constructing the density matrix through a series of column transfer matrices, Nishino and Okunishi made use of the corner transfer matrices. The CTMRG method has shown many merits for the two-dimensional classical models which can be mapped into interactionaround-face (IRF) models. Since the system can be easily extended to very large lattices, the finite size effect can be ignored. The error mainly comes from the finite dimensions of the renormalised phase space, which only becomes severe for huge lattices at or very close to the critical point. Numerically it has been observed that the extrapolation from the finite size scaling can provide very accurate information about the criticality.

We will focus on the TLIM composed of two identical layers. In this case, the model has a symmetry of inter- 
changing the spins of the two layers. This symmetry is vital to the critical behaviour of the model. A breakdown of universality due to a small symmetry breaking [17] has been observed. In order to keep this symmetry, special care should be taken in the programming. Technically, the symmetry reduces the dimensions of the transfer matrix remarkably. But the extra degeneracy will slow down the convergence of the iteration which makes the problem much more difficult compared to the one-layer Ising model.

The critical behaviour can be easily observed in various quantities, such as the rapid increase of the magnetisation, the lambda peak of the capacity, and the anomalous slowing down of convergence of the renormalisation group iterations. However, to calculate the critical exponents, one needs to locate the critical points to high precision. This is done by a careful extrapolation of the renormalisation dimension $m$.

In Section 2, the TLIM is defined and transformed into an IRF model which is suitable for the CTMRG study. In the same section, the CTMRG method particular for the considered model will be introduced. The finite-size scaling and the finite renormalisation dimension effect is studied in Section 3. At the same time the critical points are located, critical exponents of the scaling law are computed. Results for the critical exponents $\eta$ and $\nu$ can be found also in Section 3. Discussions and conclusions are given in the last section.

b. The CTMRG method for the TLIM The Hamiltonian of the model is defined by

$$
H=-J_{1} \sum_{<i, j>} s_{i} s_{j}-J_{2} \sum_{<i, j>} \sigma_{i} \sigma_{j}-\lambda \sum_{i} s_{i} \sigma_{i}
$$

where $s_{i}= \pm 1$ and $\sigma_{i}= \pm 1$ are Ising spins on two identical square lattices. For concreteness, let us say, $s_{i}$ is at the site $i$ of layer $1, \sigma_{i}$ is at the corresponding site of layer 2 which is also labelled by $i$. Then $\langle i, j\rangle$ should be understood as a nearest neighbour pair of sites either on layer 1 or layer 2 , depending on which of the spin variable, $s$ or $\sigma$, is concerned. In this paper, we only discuss the symmetric ferromagnetic case with nearest neighbour coupling $J_{1}=J_{2}=J>0$ and with interlayer coupling $\lambda=\rho J>0$.

To obtain the equivalent IRF model, two species of Ising spins, $u_{i j}= \pm 1$ and $v_{i j}= \pm 1$, are introduced for each link $\langle i, j\rangle$. The Hamiltonian (1) is generalised to

$$
H^{\prime}=-J^{\prime}\left[\sum_{<i, j>} u_{i j}\left(s_{i}+s_{j}\right)+\sum_{<i, j>} v_{i j}\left(\sigma_{i}+\sigma_{j}\right)+\rho^{\prime} \sum_{i} s_{i} \sigma_{i}\right]
$$

where $J^{\prime}$ and $\rho^{\prime}$ are defined as

$$
\begin{aligned}
J^{\prime} & =\frac{1}{2} \ln \left(e^{2 J}+\sqrt{e^{4 J}-1}\right), \\
\rho^{\prime} & =\frac{\rho J}{J^{\prime}} .
\end{aligned}
$$

irrelevant constant factor to the partition function. Inversely, if the s-spins and $\sigma$-spins are summed, one will obtain the IRF model with four u-spin interaction and four v-spin interaction around each vertex. The vertex weight of the IRF model is given by

It is not difficult to show that, by summing over all $u-$ and $v$-spins, the TLIM is recovered apart from a overall

$$
W_{a b c d}=\sum_{s, \sigma= \pm 1} \exp \left\{J^{\prime}\left[s\left(u_{a}+u_{b}+u_{c}+u_{d}\right)+\sigma\left(v_{a}+v_{b}+v_{c}+v_{d}\right)+\rho^{\prime} s \sigma\right]\right\}
$$

where $a, b, c$, and $d$ denote the four $(\mathrm{u}, \mathrm{v})$-spin pairs in the links joining to the vertex. Special weights in the boundary, which have links less than four, can be defined 
in a similar way as (5) but the values of $s$ and $\sigma$ are subject to the boundary condition. For instance, for the fixed boundary condition, the weight along the boundary, $W_{a b c}$, is defined by

$$
W_{a b c}=\exp \left[J^{\prime}\left(u_{a}+u_{b}+u_{c}+v_{a}+v_{b}+v_{c}+\rho^{\prime}\right)\right] .
$$

The vertex at the corner has a weight

$$
W_{a b}=\exp \left[J^{\prime}\left(u_{a}+u_{b}+v_{a}+v_{b}+\rho^{\prime}\right)\right] .
$$

They are schematically given in Figure 11a,b,c, respectively.

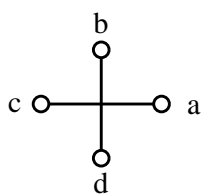

(a)

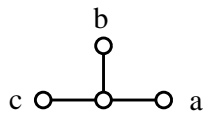

(b)

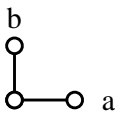

(c)
FIG. 1: (a) A full vertex weight $W_{a b c d}$; (b) an edge vertex weigh $W_{a b c} ;(\mathrm{c})$ a corner vertex weigh $W_{a b}$.

The partition function is a trace of products of all vertex weights. The trace here means to sum over all spins. In the following, whenever two vertices are connected by a link, a summation over the (u,v)-spin pair in that link is implied. The vertex weights of a quarter lattice, after summation over all inner spins and boundary spins as it has been implied, is called a corner transfer matrix $(\mathrm{CTM})$, denoted by $C_{\alpha \beta}(N)$. Here $\alpha$ and $\beta$ denote the spin configurations of two open sides of the corner respectively, and $N$ is the size of the corner. A schematic representation for a CTM is given in Figure 2 a.

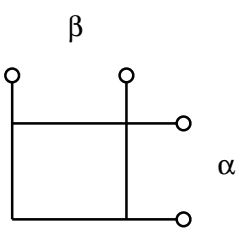

(a)

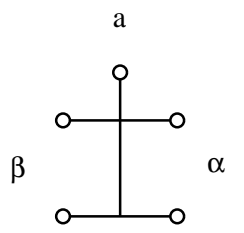

(b)

FIG. 2: (a) A corner transfer matrix (CTM), $C_{\alpha \beta}(2)$; a half-row transfer matrix (HRTM), $P_{\alpha \beta, a}(2)$.

Another matrix we need is the so-called half-row transfer matrix (HRTM) denoted by $P_{\alpha \beta, a}(N)$. It is defined by the recursion relation

$$
\begin{aligned}
P_{\alpha \beta, a}(N) & =\sum_{c} W_{a b c d} P_{\alpha^{\prime} \beta^{\prime}, c}(N-1), \\
P_{b c, a}(1) & =W_{a b c},
\end{aligned}
$$

where $\alpha^{\prime}$ and $\beta^{\prime}$ are spin configurations of two sides of the HRTM with size of $(N-1) ; c$ is the $(\mathrm{u}, \mathrm{v})$-spin pair of the top of that HRTM; $\alpha$ and $\beta$ are spin configurations of the enlarged HRTM with $\alpha$ representing $\left(\alpha^{\prime}, b\right)$ and $\beta$ representing $\left(\beta^{\prime}, d\right)$ respectively. For each value of $a$, $P_{\alpha \beta, a}(N)$ can also be considered as a matrix, denoted by $P_{a}(N)$. Figure $2 \mathrm{~b}$ is an example of the HRTM.

In the process of the CTMRG iterations, the size of CTM is enlarged by one lattice spacing each time. This is done in this way: (1) glue a HRTM to each open side of the CTM, (2) complement the bigger corner by adding a vertex weight. The recursion relation is

$$
C_{\alpha \beta}(N)=\sum_{\alpha^{\prime \prime} \beta^{\prime \prime}} \sum_{c d} W_{a b c d} P_{\alpha^{\prime} \beta^{\prime \prime}, c}(N-1) . C_{\alpha^{\prime \prime} \beta^{\prime \prime}}(N-1) P_{\alpha^{\prime \prime} \beta^{\prime}, d}(N-1) .
$$

It is schematically shown in Figure 3 .

By use of CTM, the partition function of an even size lattice is simply the trace of the CTM to power four

$$
Z_{2 N}=\operatorname{Tr}\left(C(N)^{4}\right) .
$$

Therefore, the CTM and the density matrix have common eigenvectors. Denote the eigenvalues of $C$ by $\left\{\omega_{k} \mid k=0,1,2, \ldots\right\}$, assuming that $\omega_{0} \geq \omega_{1} \geq \omega_{2} \geq$ $\ldots$, the eigenvalues of the density matrix are $\left\{\omega_{k}^{4} \mid k=\right.$ $0,1,2, \ldots\}$. According the spirit of DMRG, only a certain number of the biggest eigenstates will be kept. For instance, the first $m$ eigenstates are kept, the eigenstates with smaller eigenvalues $\left\{\omega_{k} \mid k>m\right\}$ are ignored. $m$ is the so-called renormalisation dimension.

The CTMRG iterations contains the following steps: (1) construct $C(N)$ of a small CTM which is small enough to be exactly diagonalised; (2) construct $P_{a}(N)$; (3) diagonalise $C(N)$; (4) if the dimension of $C(N)$ is smaller than $m$, all eigenstates are used as basis of the renormalised phase space, otherwise only $m$ eigenstates with the biggest eigenvalues are used as basis; (5) by use of the eigenvectors which have been chosen for the renormalised space, form a projection operator $U(N)$ that projects old spin states into the renormalised space; (6) use $U(N)$ to project $P_{a}(N)$ to the renormalised HRTM 


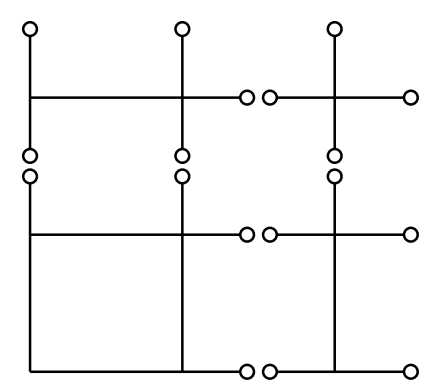

FIG. 3: Enlarging $C_{\alpha \beta}(2)$ into $C_{\alpha \beta}(3)$.

$P_{a}^{r}(N) ;(7)$ form a diagonal matrix $C^{r}(N)$ by the first $m$ largest eigenvalues, which is the renormalised CTM; (8) use $P_{a}^{r}(N), C^{r}(N)$, and the vertex weight $W$ to form a bigger CTM, $C(N+1)$, and a bigger HRTM, $P(N+1)$, and repeat the above procedure from step (3) with $N$ replaced by $N+1$.

Since the renormalised phase space has dimension $\leq$ $m$, the CTMRG in principle can infinitely go on. Therefore the lattice can be easily enlarged to very big sizes.
Particularly for the TLIM, it is efficient to choose the basis to be symmetric and antisymmetric in the exchange of two layers. In this way, the CTM is automatically block diagonal. The symmetric block and the antisymmetric block can be diagonalised separately. This is not just allowing larger $m$ and high precision, but is also essential for keeping the presumed symmetry between two layers.

For small interlayer coupling, there are many approximate degenerate eigenstates due to the layer symmetry. If one does not separately consider the symmetric and antisymmetric phase space, it is easy to destroy the layer symmetry in the renormalisation procedure.

Following the method of Nishino and Okunishi [20, the magnetisation $M$ can be calculated on an odd size lattice which is constructed by inserting a HRTM matrix between two adjacent CTM matrices and adding a proper vertex at the lattice centre. The probability that the central site has a spin state $(s, \sigma)$ is given by

$$
M^{s \sigma}=\frac{\sum_{a b c d} X_{a b c d}^{s \sigma} \operatorname{Tr}\left(P_{a}^{r} C^{r} P_{b}^{r} C^{r} P_{c}^{r} C^{r} P_{d}^{r} C^{r}\right)}{\sum_{a b c d} W_{a b c d} \operatorname{Tr}\left(P_{a}^{r} C^{r} P_{b}^{r} C^{r} P_{c}^{r} C^{r} P_{d}^{r} C^{r}\right)},
$$

where $X_{a b c d}^{s \sigma}$ is defined by

$$
X_{a b c d}^{s \sigma}=\exp \left\{J^{\prime}\left[s\left(u_{a}+u_{b}+u_{c}+u_{d}\right)+\sigma\left(v_{a}+v_{b}+v_{c}+v_{d}\right)+\rho^{\prime} s \sigma\right]\right\}
$$

The magnetisation of layer $1, M=<s>$, is

$$
M=M^{++}+M^{+-}-M^{--}-M^{-+} .
$$

Due to the layer symmetry, it is also one half of the total magnetisation. The local energy density $E$ can be calculated in a similar way.

$$
E=\frac{\sum_{a b c d} Y_{a b c d} \operatorname{Tr}\left(P_{a}^{r} C^{r} P_{b}^{r} C^{r} P_{c}^{r} C^{r} P_{d}^{r} C^{r}\right)}{\sum_{a b c d} W_{a b c d} \operatorname{Tr}\left(P_{a}^{r} C^{r} P_{b}^{r} C^{r} P_{c}^{r} C^{r} P_{d}^{r} C^{r}\right)},
$$

where

$$
Y_{a b c d}=\sum_{s, \sigma} X_{a b c d}^{s \sigma} \ln \left(X_{a b c d}^{s \sigma}\right)
$$

c. Extrapolation and finite-size scaling At the critical point $J_{c}$, it is expected that $M$ obeys the finitesize scaling form $M \sim L^{-(d-2+\eta) / 2}$, where $L$ is the lattice size, so

$$
\ln M(L)=\text { const }-\frac{d-2+\eta}{2} \ln L
$$

If we calculate $\partial \ln M / \partial \ln L$ and plot it with $\ln L$ as the horizontal axis, the curve should be a horizontal line for the critical point $J_{c}$ with the vertical value of the line as $-(d-2+\eta) / 2$. For $J$ near $J_{c}$, there should be deviations to the power-law behaviour. A simple physics consideration reveals that the curves should be in the

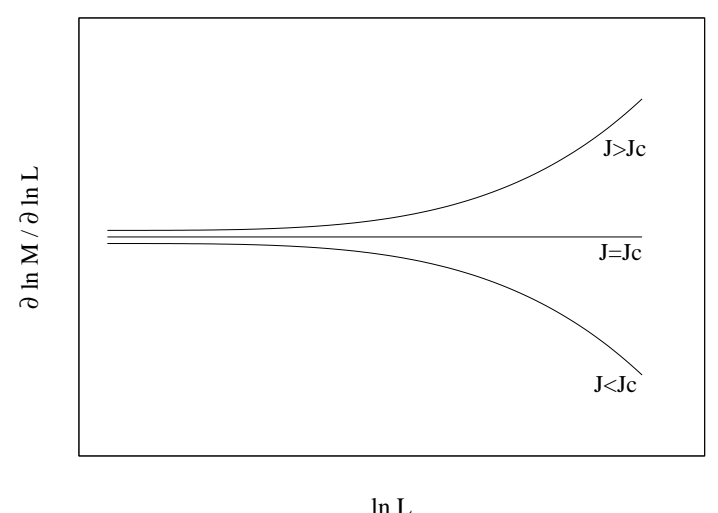

FIG. 4: Schematic plot of the ideal curves $\partial \ln M / \partial \ln L$ versus $\ln L$.

form as shown in Figure 4 . So by searching for the "best" horizontal line, one can determine the critical point $J_{c}$ as well as the critical exponent $\eta$.

Things are not so easy, however. Figure 5 shows the 


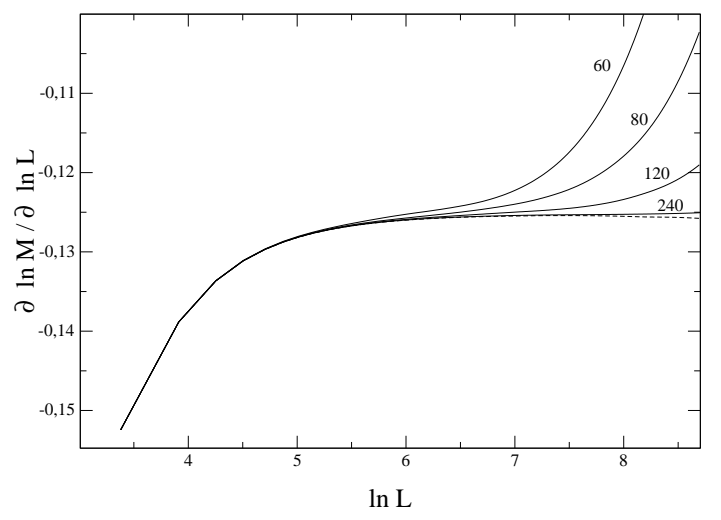

FIG. 5: $\quad \partial \ln M / \partial \ln L$ plotted versus $\ln L$ for $\rho=0.1, J=$ 0.397726 . The solid curves are for $m=60,80,120,240$. The dashed-line curve is the result of an extrapolation to $m \rightarrow \infty$.

curves of $\partial \ln M / \partial \ln L$ against $\ln L$ for $\rho=0.1, J=$ 0.397726 with different renormalisation dimensions $m$. This $J$ value is quite near to $J_{c}$. For small $L$, there exists a region where the lattice size is too small and finite-size scaling has not come into play. We will refer to this region as the small-size region. As $L$ increases, the number of states of the system increases while the renormalisation dimension $m$ remains unchanged, and we will suffer from the "finite- $m$ effect" in due time. To get a more complete knowledge of the finite- $m$ effect, let us look at Figure 6 where curves for different $J$ with $m=60$ are

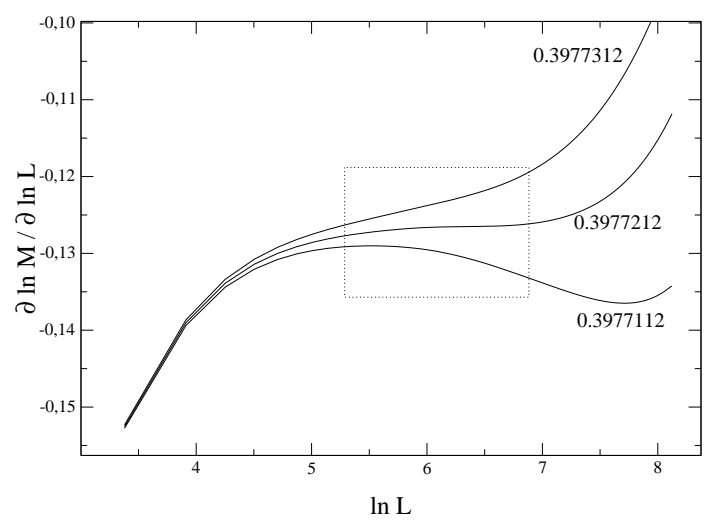

FIG. 6: $\quad \partial \ln M / \partial \ln L$ plotted versus $\ln L$ for $\rho=0.1, m=60$, for three different values of $J$.

shown. $m=60$ is relatively small in our calculation and it has a more drastic finite- $m$ effect. When $L$ is small, all the three curves shown in Figure 6 increase, which is an indication of the small-size effect. After a certain value of $L$, one of the curves begins to bend down and the other two become much flatter. Here the critical behaviour dominates. However, the finite- $m$ effect soon shows itself by raising the curves, which is also demonstrated clearly in Figure 5 . As a result, only approximately the part inside the dotted-line box resembles Figure 1. It is hard to tell exactly where the finite- $m$ effect begin to dominate without comparison with curves of higher $m$.

\begin{tabular}{r|cc}
\hline$m$ & $J_{c}^{(m)}$ & $(d-2+\eta) / 2$ \\
\hline 60 & 0.3977212 & 0.12629 \\
80 & 0.3977230 & 0.12620 \\
120 & 0.3977252 & 0.12548 \\
240 & 0.3977260 & 0.12529 \\
\hline$\infty$ & 0.3977264 & 0.12520 \\
\hline
\end{tabular}

TABLE I: The critical point and critical exponent $(d-2+\eta) / 2$ at $\rho=0.1$ estimated through first looking for the most flat curve of $\partial \ln M / \partial \ln L \sim \ln L$ for certain $m$ then extrapolating to infinite $m$.

Nevertheless, some useful information can be drawn from the analysis. If a curve bends down for a period of $L$, one can judge that it corresponds to a $J$ that is smaller than $J_{c}$. In other words, if a curve has a local maximum, it corresponds to a $J$ with $J<J_{c}$. Thus the point now is to find $J_{c}^{(60)}$, above which the corresponding curve has no local maximum and below which it has. $J_{c}^{(60)}$ can be understood as a lower limit for $J_{c}$. In general, one can obtain $J_{c}^{(m)}$ as an estimate of $J_{c}$. The former will approach the latter from below as $m \rightarrow \infty$. Meanwhile the vertical value of the horizontal part of the curve with $J=J_{c}^{(m)}$ gives the estimate for $(d-2+\eta) / 2$. To make the above discussion simpler, we have limited it only to the case with similar behaviour as that of $\rho=0.1$. Later we will see in Figure 7, that the small-size effect may

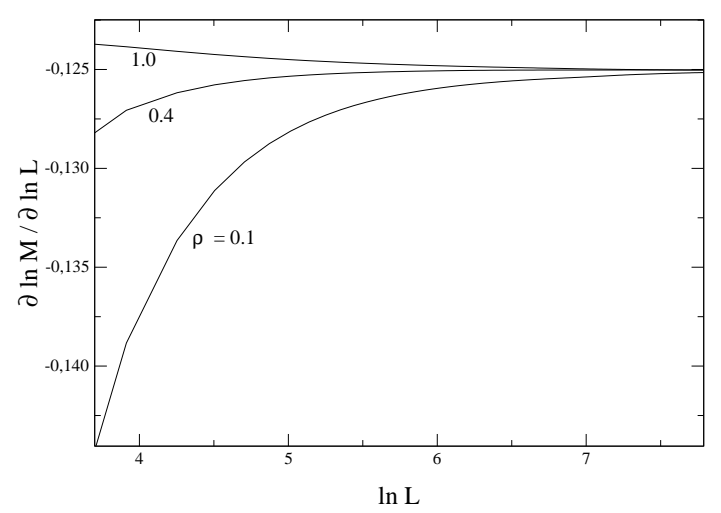

FIG. 7: $\quad \partial \ln M / \partial \ln L$ plotted versus $\ln L$ for $J=J_{c}$ with $\rho=1.0,0.4,0.1$ from above, respectively.

show itself differently as $\rho$ changes. But similar analyses can still be developed for other cases.

In most of our calculations, we chose $m$ to be 60,80 , 120 , and 240. For each $m$, we calculated the magnetisation with a set of $J$ values and then determined the critical point using the method described above. The results for $\rho=0.1$ are shown in Table 4 . The last row shows the extrapolated values for $m=\infty$.

An alternate and finer way for dealing with the finite$m$ problem is to extrapolate the data to $m \rightarrow \infty$ first and then analyse the extrapolated data. The extrapolation should lead to reasonable and smooth curves for 
$m=\infty$, and we assume the data converge monotonically as $m \rightarrow \infty$. This defines our criterion for choosing the extrapolation formula. We still take as an example the case with $J=0.397726, \rho=0.1$, whose curves are plotted in Figure 5 . For each value of lattice size $L$, we fit the points $\left(1 / m, M^{(m)}(L)\right)$, where $m=60,80,120,240$, with

$$
y=a_{5} x^{5}+a_{4} x^{4}+a_{3} x^{3}+a_{0} .
$$

$a_{0}$ then is considered to be the value for $m=\infty$ (see Figure 8). Eq.(18) does not include terms of $x^{1}$ and $x^{2}$

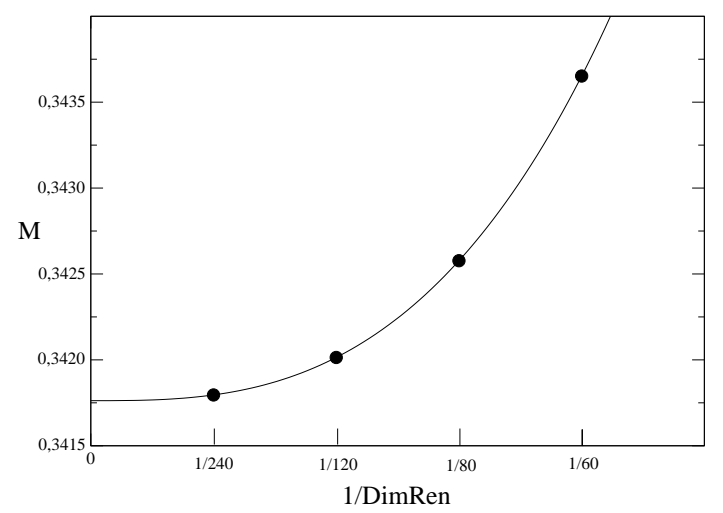

FIG. 8: Extrapolation from $m=60,80,120,240$ for $J=$ $0.397726, \rho=0.1$ and $L=2001$.

because including them violates the criteria above. The extrapolated curve is also shown in Figure as a dashed line.

Now that we obtained the curves for $m=\infty$ with different $J$, we can search for the $J$ value whose $\partial \ln M / \partial \ln L \sim \ln L$ curve is closest to a horizontal line. This $J$ value is then the estimate of $J_{c}$. In our calculation, we use interpolation technique to help locate the "best" $J$, as shown in Figure 9. The solid lines are those

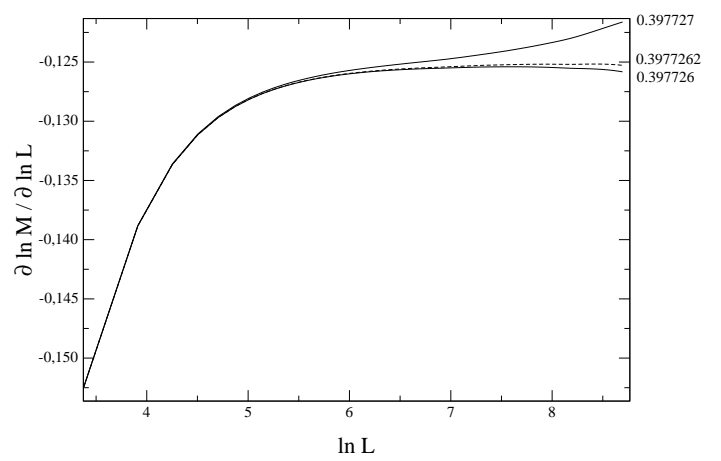

FIG. 9: $\quad \partial \ln M / \partial \ln L$ plotted versus $\ln L$ for $\rho=0.1$. The solid lines are the extropolated curves for $J=0.397726$ and $J=0.397727$. The dashed line is obtained from an interpolation between the two values of $J$.

extrapolated to infinite $m$. We can obtain the magnetisation for any value of $J$ that is between $J=0.397726$, for which the curve bends down, and $J=0.397727$, where

\begin{tabular}{r|ccc}
\hline$\rho$ & $J_{c}^{(\infty)}$ & $(d-2+\eta) / 2$ & $d-\frac{1}{\nu}$ \\
\hline 0.1 & 0.3977262 & 0.12507 & 1.0014 \\
0.4 & 0.3541412 & 0.12503 & 1.0001 \\
1.0 & 0.3117577 & 0.12502 & 0.9996 \\
\hline
\end{tabular}

TABLE II: Critical point $J_{c}$, critical exponents for $\rho=$ $0.1,0.4$, and 1.0, estimated through first extrapolating the curve of $\partial \ln M / \partial \ln L \sim \ln L$ for infinite $m$ then looking for the most flat curve.

the line bends up, by linear interpolation. We found $J_{c}$ to be 0.3977262 with $(d-2+\eta) / 2=0.12507$.

The small-size effect for different ratios is presented in Figure 7. For small $\rho$, this effect is big. In the case of $\rho=0.1$, for instance, the small-size effect is visible with $L$ up to 800 . However, it seems that we overcome the small-size effect with $L=2000$.

Having $J_{c}$ in hand, we can determine another critical exponent $\nu$ through the finite-size scaling form of the energy density

$$
E(L)-E_{\infty} \sim L^{-\left(d-\frac{1}{\nu}\right)} .
$$

The critical point $J_{c}$, critical exponents $(d-2+\eta) / 2$ , and $d-\frac{1}{\nu}$ for $\rho=0.1,0.4$, and 1.0 are collected in Table II.

We also estimated the shift exponent by taking $\rho=$ 0.005 and 0.01 . The critical points are found to be $J_{c}=0.432507$ and 0.428593 respectively, which lead to a value 1.773 for the shift exponent that consists with the theoretical prediction 1.75 with $1.3 \%$ deviation.

d. Discussion and conclusions Our numerical results suggest that the TLIM model is in the Ising universality class. That is, for a finite interlayer coupling, the critical behaviour of the TLIM is the same as that of the one-layer system. Both $\langle s\rangle$ and $\langle\sigma\rangle$ are order parameters in the transition. Our observations strongly exclude a phase transition for the electric order, except at the decoupling point $\rho=0$.

The critical line of the TLIM is in fact not a fixed line since the interlayer coupling is a relevant interaction which should flow to infinity in renormalisation group transformations. Then the TLIM resembles the one-layer Ising model in the long-range critical behaviour. To confirm this picture, we also carry out the real-space renormalizational group transformation to the model. The lattice is divided into $3 \times 3$ blocks. All nearest neighbour interactions are kept. The RG trajactories are plotted in Figure 10. Two attractive fix points both having infinite $\rho$ are observed. They are the ordered and disordered fix point, respectively. The critical line seperates two attractive fix points and obviously it is not a fix line.

However, as the interlayer coupling is small, it needs a large scale transformation to reach the infinite fixed points, so the model has extraordinary large finite-size effects as we have observed in the numerical results. 


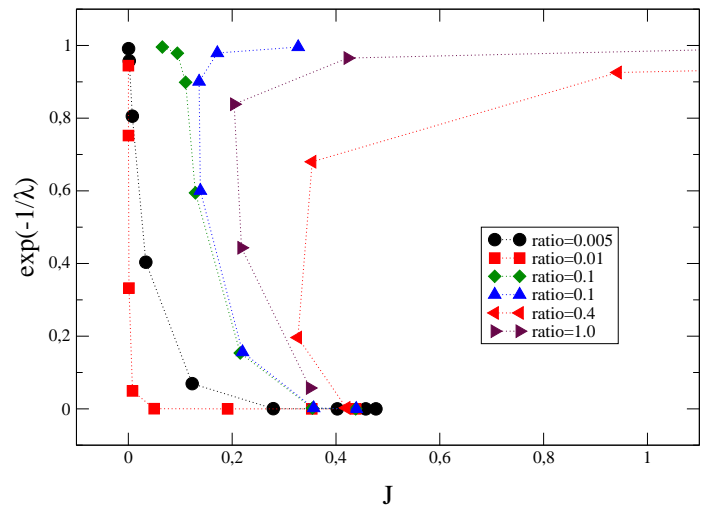

FIG. 10: The RG trajectories mapped to the TLIM parameter space are plotted. The vertical axis is $\exp (-1 / \lambda)$ with $\lambda=\rho J$. Corresponding to the curves in the order of the legend, the starting couplings are $J=0.477,0.438,0.438,0.439$, 0.42 , and 0.35 , respectively.

The decoupling limit shows singularities due to the ex- istence of the severe competition between the unstable decoupling fixed point and the stable infinite interlayer coupling fixed points which, however, are far away. This supported in some sense by the unusual sensitivity of the shift exponent on the couplings [16, 17] and the strange layer symmetry breaking in the effective state at the decoupling limit 14. Our estimate for the shift exponent is consistent with the theoretical prediction.

The unusual small-size effects will have important consequences on the local properties of the TLIM. It is manifested in the short-time critical behaviour that we recently observed in Monte Carlo simulations which will be reported elsewhere [19].

\section{Acknowledgments}

The authors thank B. Zheng for helpful discussions.
[1] L. E. Ballentine, Physica 30 (1964) 1231

[2] G. A. T. Allan, Phys. Rev. B 1 (1970) 352

[3] R. Abe, Prog. Theor. Phys. 44 (1970) 339

[4] M. Suzuki, Prog. Theor. Phys. 46 (1971) 1054

[5] K. Binder, Thin Solid Films 20 (1974) 367

[6] J. Oitmaa and G. Enting, J. Phys. A 8 (1975) 1097

[7] H.E. Oepen, M. Benning, H. Ibach, C.M. Schneider, and J. Kirschner, J. Magn. Magn. Mater. 86 (1990) L137

[8] K. Shimazaki, S. Ohnuki, H. Fujiwara, and N. Ohta, J. Magn. Magn. Mater. 104-107 (1992) 1017

[9] Z. B. Li, L. Schülke, and B. Zheng, preprint (1994) Univ.GH Siegen

[10] T. Horiguchi, A. Lipowski, and N. Tsushima, Physica A 224 (1996) 626

[11] J. Wosiek, Phys. Rev. B49 (1994) 15023

[12] L. Angelini, D. Caroppo, M. Pellicoro, and M. Villani, J. Phys. A: Math. Gen. 25 (1992) 5423

[13] L. Angelini, D. Caroppo, M. Pellicoro, and M. Villani, Physica A 219 (1995) 447
[14] L. Angelini, D. Caroppo, M. Pellicoro, and M. Villani, Physica A 237 (1997) 320

[15] A. Lipowski and M. Suzuki, Physica A 198 (1993) 227

[16] A. Lipowski and M. Suzuki, Physica A 250 (1998) 373

[17] T. Horiguchi and N. Tsushima, Physica A 238 (1997) 295

[18] C. Hu, N. S. Izmailian and K. B. Oganesyan, condmat/9904008

[19] Z. B. Li, H.J. Luo, L. Schülke, and Q. Wang, preprint

[20] T. Nishino and K. Okunishi, J. Phys. Soc. Jpn. 65 (1996) 891

[21] T. Nishino, K. Okunishi, and M. Kikuchi, Phys. Lett. A 213 (1996) 69

[22] T. Nishino and K. Okunishi, J. Phys. Soc. Jpn. 66 (1997) 3040

[23] S. R. White, Phys. Rev. Lett. 69 (1992) 2863

[24] S. R. White, Phys. Rev. B 48 (1993) 10345 